\title{
The Effects of Interfacial Tension and Spreading on Relative Permeability in Gas Condensate Systems
}

\author{
Munkerud P. K, Torsaeter $\mathbf{0}$.
}

\author{
IKU, Norway
}

\begin{abstract}
Copyright 1985, Steering Committee of the European IOA - Symposium.
This peper was presented at the 8th. European IOR - Symposium in Vienna. Austria, May 15 - 17, 1985

This paper was selected for presentation by the Steering Committee, following review of information contained in an abatract

aubmitted by the authorls;. The paper, os presented has not been neviewed by the stoering Committer.
\end{abstract}

\begin{abstract}
In this paper the results of a series of flow experiments performed on a gas condensate system will be presented. The objective of the experimental study was to establish the effects of interfacial tension between gas and corresponding liquid (in presence of water) on relative permeability for a gas condensate system in the two phase region below the dew-point pressure. The experiments were performed at conditions that gave the desired phase behaviour at relatively moderate pressure and temperature for the fluid system which consisted of the 6 components methane, ethane, propane, pentane, hexane and decane. This system gave reasonably low interfacial tensions and relatively high liquid drop-outs at moderate pressure. As a reference systems were used a two-component system of nitrogen and decane, and a threecomponent system of ethane, propane and butane. The latter systems should give the extreme cases of medium to high interfacial tension which could be measured readily both by dynamic displacement method and by steady state method. In the attempt to change the spreading characteristics of the fluid systems butanol was added to the water phase, but no distinct non-spreading was achieved for neither the 6-component system nor the reference systems. The applied porous medium was Berea sandstone (50 $\mathrm{cm}$ long and water wet). This porous medium
\end{abstract}

has a reasonably wide pore size distribution which would promote two-phase production of fluid from the core during dynamic displacement. All experiments were performed with an immobile water saturation.

The experiments showed that when the interfacial tension approached zero, the relative permeabilities to both liquid and gas became straight lines. The residual and critical saturations were both close to zero as the IFT approached zero. In the case of spreading, the residual and critical saturations were not significantly smaller than for the non-spreading case even when the IFT was high. The relative permeability was considerably more influenced by the IFT between gas and condensed liquid than by spreading characteristics.

\section{Introduction}

The objective of the experimental study was to establish the effects of interfacial tension between gas and corresponding liquid and spreading (in presence of water) on relative permeability for a gas condensate system in the two phase region below the dew-point pressure. The measurements of relative permeability for the 6-component gas 
condensate model system were performed at reservoif conditions in a $0.5 \mathrm{~m}$ long Berea core.

To investigate relative permeability of gas condensate fluids at different flow conditions, a six component model system was designed, exhibiting the typical behaviour of condensates. The designed system had a maximum liquid drop out of $30.1 \%$ at $18.2 \mathrm{MPa}$ and $41^{\circ} \mathrm{C}$. The interfacial tension of the fluid system at different pressures was measured by surface light scattering technique ${ }^{2}$, while the IFT for the reference systems were calculated by the Parachor method. ${ }^{3,4}$ The relative permeability of the gas condensate system in the Berea core was studied by the following types of experiments (below the dew point pressure) at different pressures (interfacial tensions) in vertically mounted core:

- semi steady state flow;

- displacement of liquid by equilibrium gas.

None of these are standard experiments and therefore the equipment and experimental procedures had to be specifically designed and developed for the purpose. In the following chapters some published work on the topic will be discussed, and the applied experimental equipment and procedures will be described. The results will then be presented and discussed with references to tables and figures. Finally, the conclusions will be drawn (emphasis on the utility of the methodology in reservoir evaluation).

\section{BACKGROUND}

The general equations describing the flow of two or more phases show that the flow rate in a porous medium is a function of the absolute permeability, relative permeability to the fluids involved, fluid viscosities, pressure gradient along the core and gravity. Relative permeabilities depend on other factors as well. Some of these are the ratio of viscosities, $\mu_{2} / \mu_{1}$; the ratio of gravity to capillary forces (Bond number), $N_{B}=\left[\left(p_{2}-\rho_{1}\right) g R^{2}\right] / \sigma$; the ratio of inertial forces to viscous forces (Reynolds number), $\operatorname{Re}=\left(\rho_{1} u \sqrt{k}\right) / \mu$; the ratio of viscous to capillary forces (capillary number), $\mathrm{N}_{\mathrm{C}}=\left(\mu_{1} \mathrm{u}\right) / \sigma$; and wettability. When two or more phases exist, exchanges between phases can modify the physical and chemical properties of the fluids, especially at the interfaces. For values of $\mathrm{N}_{\mathrm{c}}$ in the range $10^{-6}$ $10^{-4}$, the influence of the capillary forces is not negligible. The decrease in interfacial tension causes the $S_{\text {or }}$ to decrease and approach 0 , i.e. increase in oil recovery. Usually the endpoint relative permeability to oil is closely dependent on the capillary number, especially when the value of $\sigma$ is small.

Bardon and Longeron ${ }^{5}$ investigated the influence of very low interfacial tensions on relative permeability. In their study, they used binary hydrocarbon mixtures with liquid and vapour phases at equilibrium at the experimental conditions. The level of interfacial tension was controlled by varying the equilibrium pressure and thus the composition of the mixtures. In each case, the two phases were separated after phase equilibrium had been achieved. The porous medium was saturated with the liquid phase, which then was displaced by the vapour phase with a very small pressure drop across the core to disturb the phase equilibrium as little as possible.

The experimental velocities were sufficiently low to secure laminar flow. For low-permeability porous media, the influence of the Bond number, $N_{B}$, is negligible, since gravity has very little effect on the shape of the interfaces between the two hydrocarbon phases.

The relative permeabilities were calculated by the Welge method. An examination of the relative permeability curves obtained, which also were partially validated by numerical simulation, showed that the shape of the curves varied considerably depending on the physical properties of the phases.

A classification made in terms of $\sigma$, the interfacial tension, showed that the major change in relative permeabilities seemed to occur in the vicinity of $\sigma$ $=0.05 \mathrm{mN} / \mathrm{m}$. Above this value, and for a given porous medium, the relative permeability curves to be used in numerical simulation can be handled with reasonable accuracy by traditional techniques.

For very low values of interfacial tension, though, especially close to the critical point, prediction is more difficult, since there are great changes in the relative permeabilities for small changes in interfacial tension. For values of $\sigma=0.001 \mathrm{mN} / \mathrm{m}$, the relative permeabilities can be represented by two straight lines with a slope of one in the linear relative permeability normalized saturation diagram.

The integrated experimental methodology presented by Borbiaux et al. ${ }^{6}$ proved to be efficient 
for measurement of gas condensate flow properties at various pressure and interfacial tension conditions. The method benefits from the combination of in situ saturation measurements, visual observation of core effluents, and onstream measurements of liquid drop-out and gas viscosity. A main conclusion from the work is that they obtained low and reproducible residual and critical saturations for low IFT equilibrium gas injection $(0.03 \mathrm{mN} / \mathrm{m})$ which could be correlated to the total residual liquid saturation (water and condensate). It is also claimed that the steady state technique can not be used since the method accumulates condensate in the core before break through.

Regarding the effects of IFT on relative permeability it seemed to effect the relative permeability of the liquid more than that of the gas but this conclusion needs to be more investigated.

Asar and Handy ${ }^{7}$ performed measurements of the influence of interfacial tension on gas-liquid relative permeability in a gas condensate system. They found that the curves of the individual relative permeabilities ( $k_{r g}$ and $k_{r l}$ ) vs. gas saturation, tended to approach straight lines as IFT approached zero. The relative liquid permeability decreased more rapidly than the relative permeability to gas with increasing IFT. Residual gas and liquid saturations were higher with higher IFT. The gas saturation at which the gas and liquid relative permeability curves intersected, was higher as the IFT decreased, indicating a decrease in the oil-wet character of the system. The level of $\mathrm{k}_{\mathrm{rl}}$ and $\mathrm{k}_{\mathrm{rg}}$ at which these two curves intersected was higher for lower IFT values.

Saturation history effects at intermediate IFTs were investigated and appeared not to be significant in this type of experiment. Relative permeability results obtained at the higher IFT level ( $\sigma$ $=0.83 \mathrm{mN} / \mathrm{m}$ ) approached results obtained for a nitrogen/kerosene flood, even though the nitrogen/kerosene IFT was much higher. The relative gas and liquid permeabilities for gas-condensate reservoirs appear to correspond to those for normal gas/oil systems except at near critical conditions.

Saeidi and Handy ${ }^{8}$ have reported an investigation of flow and phase behaviour of gas condensate and volatile oils in porous media In their work, gas and liquid permeabilities were measured for gas condensate systems at high pressure. Similar measurements were made on bubble point systems of different volatility close to atmospheric pressure. Comparison between these two systems indi- cated that gas condensate relative permeability values were lower in the region of low to intermediate liquid saturation than the comparable values for the bubble point systems. Furthermore, gas condensate relative permeability ratios indicated a lower critical liquid saturation for flow than that of the bubble point systems.

Laboratory phase behaviour studies on hydrocarbon binary mixtures flowing through a porous medium, were performed to test the suitability of PVT relationships obtained from steady state PVT cells in prediction of condensate reservoir fluid behaviour under actual flow conditions. The results obtained in the work indicated that at lower laboratory pressure depletion rates (still much greater than field rates) equilibrium was maintained during transient flow of the hydrocarbon binary mixtures used. At higher pressure depletion rates some non equilibrium behaviour was observed, particularly during the revaporization period.

It is well known that in gas displacement processes will gravity forces play an important role in the oil and condensate recovery by so called gravity drainage, which occurs in permeable reservoirs, saturated with near critical fluids such as volatile oils and gas condensates. In such cases, the saturation profiles can be interpreted as reflecting equilibrium between gravity and capillary forces. Very low residual liquid saturation can be obtained just behind the displacement front.

Dumore and Schols ${ }^{9}$ pointed out that the residual oil saturation of very permeable sandstones in presence of connate water, could be extremely low (4 PV\%) in gravity stable gas injection processes. They also showed that low oil saturation could be obtained in gravity drainage experiments after long drainage times in sand columns. These low values of residual oil saturation obtained by film flow, were independent of whether or not the oil phase spreads on water in presence of gas.

The high gravity drainage performance was clearly recognized by King and Stiles ${ }^{10}$ in their field study of the East Texas Hawkins reservoir ( $87 \%$ recovery). More recently, it was observed by Hagoort ${ }^{11}$ that the oil relative permeability was the key factor in the gravity drainage process.

Delclaud et al. ${ }^{12}$ have presented a series of unsteady-state gas displacement experiments in homogeneous and fairly permeable core samples. The displacements were performed with an analysis of 
transient phenomena by measurement of pressure and saturation ( $\mathrm{X}$-ray attenuation) along the entire length of the core. From these local measurements, it was pointed out that the capillary pressure/saturation relationship, recorded during gasflooding, was identical to the one determined by the restored-state method

The differential flowing pressure did not affect the direct permeability measurements since the higher the differential flowing pressure, the wider was the desaturation zone. Experimental results obtained with various differential pressures were correctly matched by a numerical model which always used the same relative permeability and capillary pressure curves as input data. This model took into account capillary phenomena and observed the following boundary conditions: very low gas saturation at the outlet face and no liquid pressure gradient at the inlet face.

Delclaud et al. ${ }^{12}$ investigated also gas/oil relative permeabilities for high permeability oil reservoir application by unsteady-state displacement tests. They found that gas-oil relative permeabilities were unchanged when the interfacial tension between the phases varied in the range $0.6-30 \mathrm{mN} / \mathrm{m}$. For the type of porous medium used (very permeable sandstone), very low residual oil saturations were found: $3.5-6 \mathrm{PV} \%$, corresponding to $\mathrm{k}_{\mathrm{ro}}=10^{-}$ 5 , which was a practical lover limit for significant flow.

\section{EQUIPMENT AND PROCEDURES}

Fluid system. Based on preliminary simulations on the compositional simulator COPEC ${ }^{15}$, a six component gas condensate fluid system consisting of methane, ethane, propane, pentane, hexane and decane, was designed. The system showed pronounced retrograde condensation at moderate pressures and temperatures, and had properties with respect to composition, density etc. that were similar to real gas condensate systems. The measurements of the PVT properties of the fluid included: CME (constant mass or composition expansion); CVD (constant volume depletion); IFT-; density and viscosity measurements. The results of the CVD are given in Figure. 2. Interfacial tensions were measured by the surface light scattering technique ${ }^{2}$ but was also calculated by the Parachor technique as described by Hough and Stegemayer ${ }^{3}$. The liquid viscosities were measured by a modified Ruska Rolling Ball Tensiometer. Gas viscosity was calculated by COPEC ${ }^{15}$. Densities were measured by a High Pressure Anton Paar Densitometer.

Relative permeability. The relative permeability of the gas condensate system in Berea was studied by the following types of experiments at different pressures below the dew point pressure (interfacial tensions) in vertically mounted (exp. 4-6) core:

- semi steady state flow;

- displacement of liquid by equilibrium gas.

During the measurements mass balance was recorded by measuring the production of gas into a vacuumed tank and by measuring the mass of produced liquid collected in glass separators. The corresponding compositions of both phases were measured at appropriate times by gas chromatography. Pressure could be measured at 8 different points along the core if desired, making possible measurement of the total pressure gradient along the core as well as the local gradients if the $\Delta P$ was high enough. The core holder and corresponding key equipment are shown schematically in Figure 1 below.

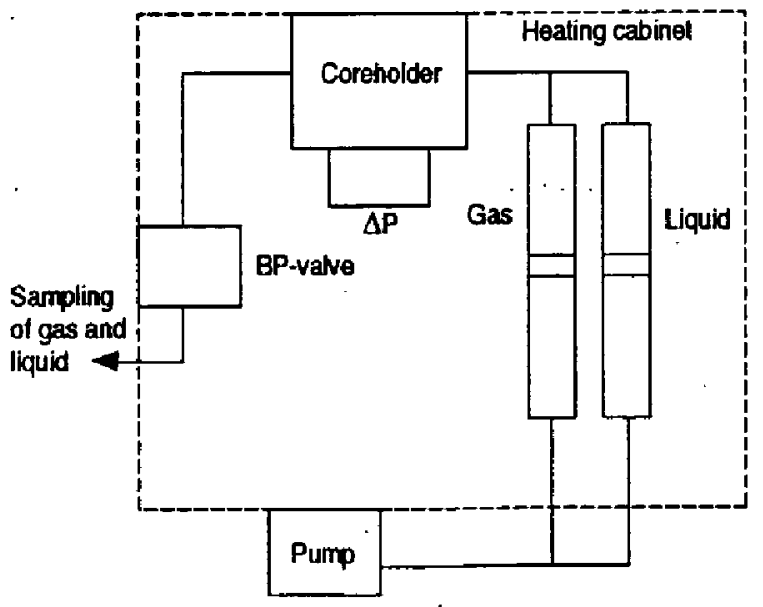

Figure I Schematic of apparatus used for displacement and steady state experiments.

Relative permeability by semi steady state flow. The semi steady state flow experiments were performed according to the following procedure (preliminary steps not included):

1. The core $(\mathrm{L}=50 \mathrm{~cm}, \mathrm{D}=5.0 \mathrm{~cm})$ was saturated with gas condensate by flushing the core containing hexane with three pore volumes of gas condensate at the determined pressure for the 
experiment (below the dew point pressure with corresponding interfacial tension). The assumption was that the composition in the liquid now saturating the core, should be equal to the liquid in the corresponding CVD experiment in which IFT-values were measured. Before the experiment was started all dead volume around the core holder were flushed with injection gas.

2. The semi steady state experiment was then started by injecting simultaneously equilibrium liquid and gas in gassliquid ratios of $0,0.2,0.4$, $0.6,0.8$ and $\infty$. The total flow rate was approximately $5 \mathrm{ml} / \mathrm{h}$ for all flow steps.

3. The $\Delta P$ was measured over a distance of $45 \mathrm{~cm}$ with a high accuracy $\Delta P$ transducer. When the change in $\Delta P$ was less than 0.01 mbar across the core, steady state was anticipated. At this point approximately $3 \mathrm{PV}$ of gas and liquid had been injected in most experiments.

4. The produced liquid was collected in glass separators and the composition of the gas passing through the separators, was analyzed automatically on GC every 35 minutes. 1-2 manual samples were collected in-between the automatic samples. The amount of gas produced, was measured by producing the gas into an evacuated tank and reading the tank pressure. The corresponding number of moles of gas was then calculated by the ideal gas law. The total number of moles produced, were calculated based on the produced gas and liquid volumes, mass and composition.

5. From production and pressure data, the average flow rate in the core was calculated. At semi steady state conditions these rates should be approximately equal to the injection rates. The viscosities of the flowing fluids were calculated from correlations based on compositional data from CVD and core production. The average liquid saturation in the core was determined from the injection and production data. The calculated relative permeability results are plotted vs. average liquid saturation in Figures 4 and 5.

After all flow steps were finished, the core was flushed with several pore-volumes of hexane and prepared for the next IFT-step.

The semi steady state experiment was used on experiments 1, 2, 4 and 5, while the displacement technique was used for experiments 3 and 6 (se Table 2 for details).

Relative permeability by displacement. The dynamic displacement experiments were performed by displacing condensate with gas in equilibrium with the condensate at the displacement pressure of 18.2 MPa (exp. 3 and 6). The displacements started with the core initially saturated with $25 \%$ immobile water and $75 \%$ hexane. The hexane was displaced by 3 pore volumes of condensate in equilibrium with gas at the displacement pressure. The core holder was connected as shown in Figure 1 (similar to semi steady state experiments).

The displacements were then performed in the following stepwise manner:

1. The tubing around the core was flushed with injection gas and the back-pressure valve adjusted to the specific pressure.

2. When the desired pressure was reached, the displacement was started with an injection rate of approximately $5 \mathrm{ml} / \mathrm{h}$.

3. The $\Delta \mathrm{P}$ was measured over a distance of $45 \mathrm{~cm}$ with a high accuracy $\Delta P$ transducer and when the rate of liquid production was less than 0.1 $\mathrm{ml} / \mathrm{h}$ from the core, the injection was stopped. At this point approximately 3 PV of gas had been injected.

4. The produced liquid was collected in glass separators and the composition of the gas passing through the separators, was analyzed automatically on GC similarly as described above. The amount of gas produced, was measured as described above, and the corresponding number of moles of each gas component was calculated by the ideal gas law. The total number of moles produced, were calculated based on the produced gas and liquid volumes, mass and composition. A phase detection system (infrared light diode for refraction measurements) detected the breakthrough of the gas and gave also a fairly accurate reading of the flow rate of each phase in the two-phase region.

5. From production and pressure data, the effective flow rate at the core outlet was calculated as well as the apparent viscosity. The viscosities of the flowing fluids were calculated from correlations based on compositional data from CVD and the core production. The average liquid saruration in the core was determined 
from the injection and production data. The relative permeabilities were calculated according to the Welge method ${ }^{1}$. The calculated relative permeability results are plotted vs. average liquid saturation in Figures 4 and 5.

6. After each displacement, the core was filled with hexane and then another experiment was performed. Note that only the lowest pressure was measured by the displacement technique.

\section{EXPERIMENTAL RESULTS}

Basic fluid and core properties. The dew point pressure of the six component gas condensate system was determined by CVD to be $19.61 \mathrm{MPa}$ at $41{ }^{\circ} \mathrm{C}$. The maximum liquid drop-out was 30.1 $\%$ of initial gas volume at $18.20 \mathrm{MPa}$. The liquid drop-out vs. pressure is plotted in Figure 2. Values for a CME-process is also given.

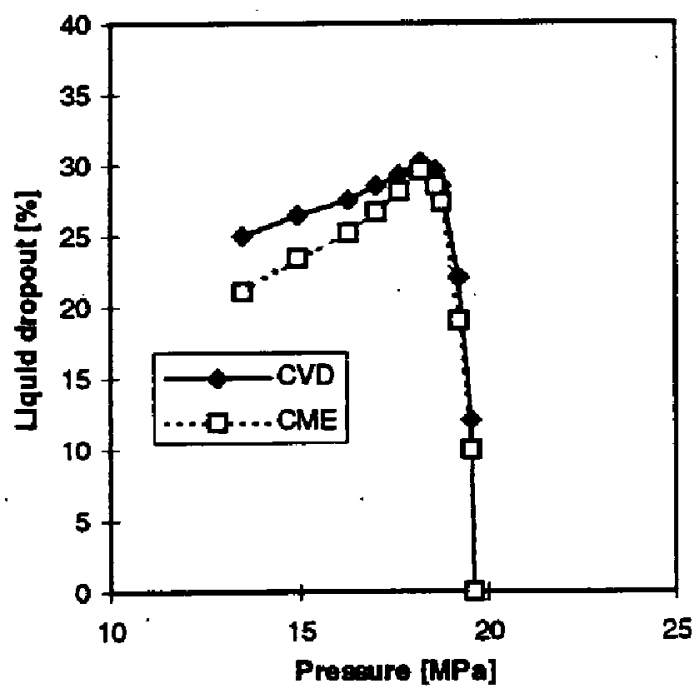

Figure 2 Liquid dropout vs. pressure in 6-component gas condensate system during $C V D$ and CME processes.

The IFT was measured during a CVD process. The obtained results of these measurements are given in Figure 3.

The properties of the Berea core are given in Table 1 below. The porosity was measured to be $15.3 \%$ and the absolute permeability to be $220 \mathrm{md}$ at experimental conditions. When irreducible water was present $\left(S_{w i}=25 \%\right)$, the permeability was reduced to $155 \mathrm{md}$.

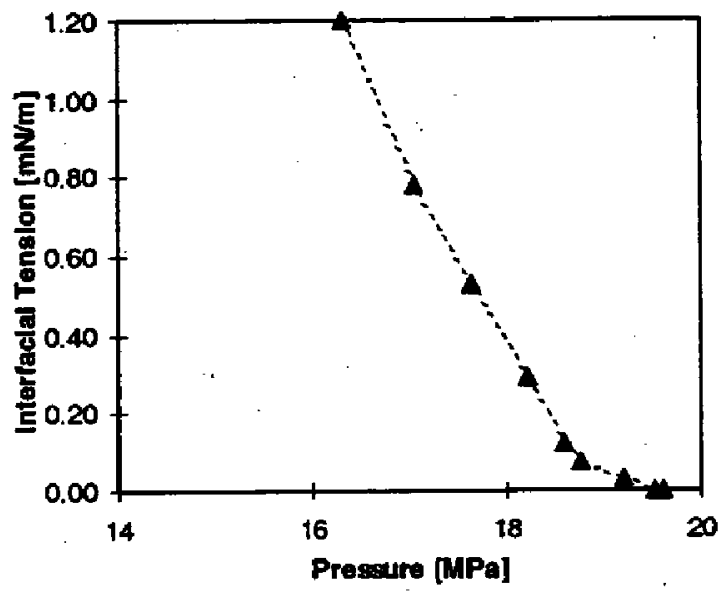

Figure 3 Interfacial tension vs. pressure for 6-component gas condensate system.

Table 1 Core properties.

\begin{tabular}{|l|c|}
\hline Length $[\mathrm{cm}]$ & 50.0 \\
\hline Diameter [cm] & 5.08 \\
\hline Distance for $\Delta \mathrm{P}[\mathrm{cm}]$ & 45.0 \\
\hline Pore volume [cm $]$ & 155 \\
\hline $\begin{array}{l}\text { Absolute perm. }\left(41^{\circ} \mathrm{C}, 20 \mathrm{MPa}\right)[\mathrm{mD}] \\
\mathrm{S}_{\mathrm{w}}=0 \%\end{array}$ & 220 \\
\hline $\begin{array}{l}\text { Absolute perm. }\left(41{ }^{\circ} \mathrm{C}, 20 \mathrm{MPa}\right)[\mathrm{mD}], \\
\mathrm{S}_{\mathrm{wi}}=25 \%\end{array}$ & 155 \\
\hline
\end{tabular}

Table 2 Key gas and liquid properties at applied pressure and temperature conditions.

\begin{tabular}{|c|c|c|c|c|}
\hline $\begin{array}{c}\text { Exp. } \\
\text { no }\end{array}$ & $\begin{array}{c}\text { Pressure } \\
{[\mathrm{MPa}]}\end{array}$ & $\begin{array}{c}\text { IFT } \\
{[\mathrm{mN} / \mathrm{m}]}\end{array}$ & $\begin{array}{c}\mu_{\text {gas }} \\
{[\mathrm{CP}]}\end{array}$ & $\begin{array}{c}\text { Mliquid } \\
{[\mathrm{cP}]}\end{array}$ \\
\hline 1 & 19.20 & 0.04 & 0.0289 & 0.0503 \\
\hline 2 & 18.60 & 0.14 & 0.0252 & 0.0573 \\
\hline 3 & 18.20 & 0.26 & 0.0234 & 0.0634 \\
\hline 4 & 19.26 & 0.03 & 0.0280 & 0.0500 \\
\hline 5 & 18.65 & 0.12 & 0.0250 & 0.0570 \\
\hline 6 & 18.15 & 0.29 & 0.0231 & 0.0636 \\
\hline
\end{tabular}

Relative permeability. The resulting relative permeabilities of both semi steady state and dynamic displacement are plotted in Figures 4 and 5 .

The calculation of relative permeability by displacement is based on the fundamental principles of the Welge method as described originally by 
Jones and Rozelle and later used by Munkerud in a previous paper ${ }^{1}$.

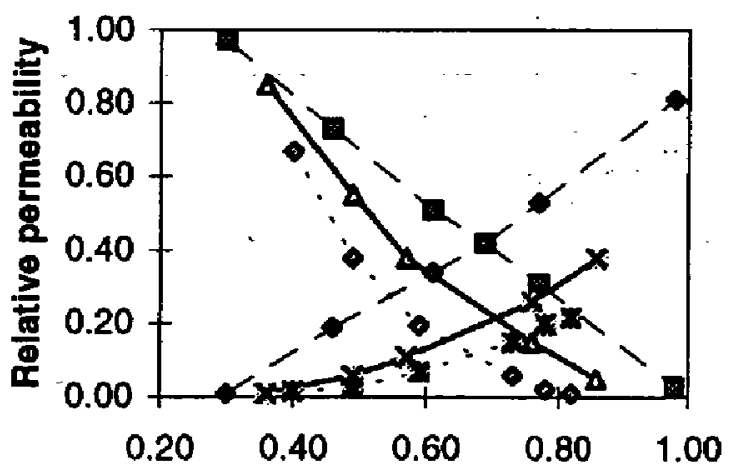

Liquid saturation

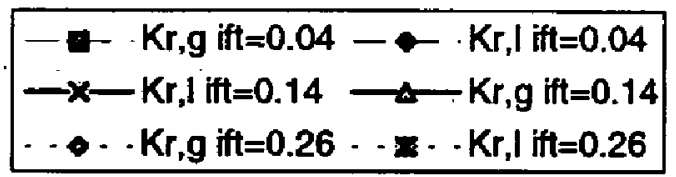

Figure 4 Relative permeability vs. liquid saturation for different interfacial tensions in a 6 component gas condensate system, Berea core.

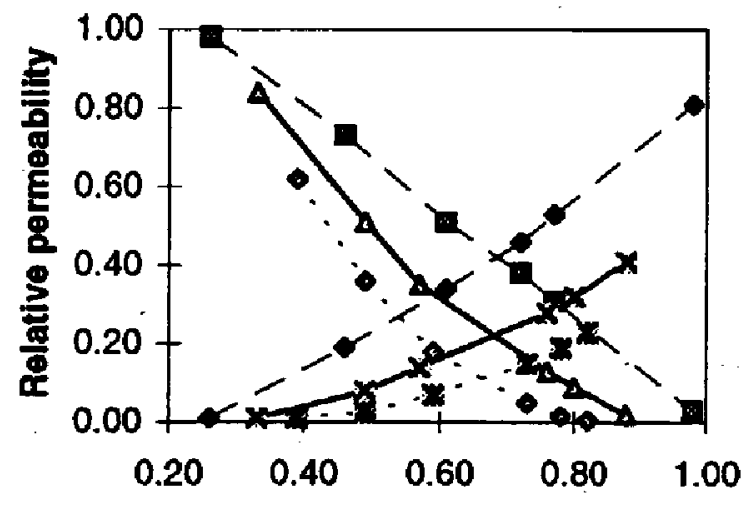

Liquid saturation

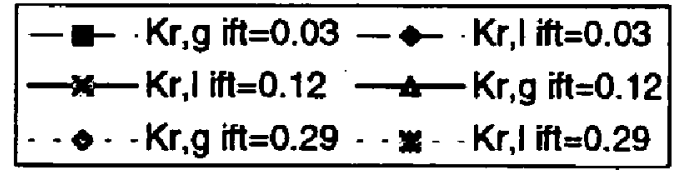

Figure 5 Relative permeability vs. liquid saturation for different interfacial tensions in a 6-component gas condensate system. Berea core.

Only two experiments are based on displacement. This is the two interfacial tensions furthest away from the dew point pressure: experiments 3 and 6 with conditions as follows: $P_{3}=18.20 \mathrm{MPa}, \mathrm{IFT}_{3}$ $=0.26 \mathrm{mN} / \mathrm{m}$; and $\mathrm{P}_{6}=18.15 \mathrm{MPa}$, $\mathbb{F T}_{6}=0.26$ $\mathrm{mN} / \mathrm{m}$.
For the semi steady state experiments the pressures and interfacial tensions were as follows: $P_{1}=19.20$ $\mathrm{MPa}, \quad$ IFT $_{1}=0.04 \quad \mathrm{mN} / \mathrm{m} ; \quad P_{2}=18.60 \quad \mathrm{MPa}$, IFT $_{2}=0.14 \mathrm{mN} / \mathrm{m} ; \quad \mathbf{P}_{4}=19.26 \mathrm{MPa}, \quad$ IFT $_{4}=0.03$ $\mathrm{mN} / \mathrm{m}$; and $\mathrm{P}_{5}=18.65 \mathrm{MPa}, \mathrm{IFT}_{5}=0.12 \mathrm{mN} / \mathrm{m}$.

The reference system with ethane, propane and butane was measured by the steady state technique and the system of decane and nitrogen was measured by dynamic displacement. The resulting relative permeability curves are given in Figure 6 below. In this case the liquid saturation is normalized $\left(S_{\mathrm{mi}}=25 \%\right)$. Since this is just for reference, all details are not given in this paper.

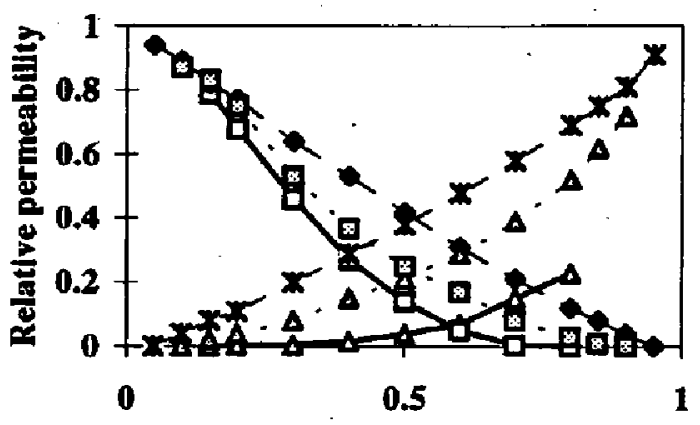

Normaliced liquid saturation

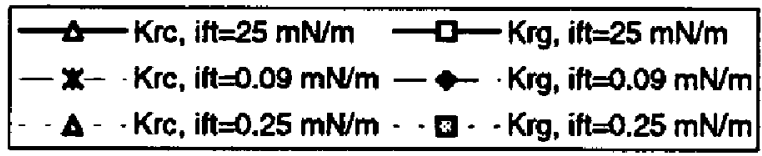

Figure 6 Relative permeability vs. normalized liquid saturation for different interfacial tensions in a three component "gas condensate" system and a nitrogendecane reference system, $I F T=25 \mathrm{mN} / \mathrm{m}$.

\section{DISCUSSION}

Fluid and core properties. The determination of the dew point pressure for the gas condensate system is regarded fairly accurate since the same dew point (19.61 $\mathrm{MPa}$ at $41{ }^{\circ} \mathrm{C}$ ) has been observed several times in different depletion experiments in condensate cells, slim tube and cores'.

As can be seen from Figure 2 the amount of liquid produced from the gas during the CVD, increased sharply from zero at the dew point pressure, to a maximum of $30.1 \%$ of initial gas volume at 18.20 $\mathrm{MPa}$. This indicates clearly the sensitivity of the system to small changes in pressure. Conse- 
quently, caution must be paid during the experiments to avoiơ úndésired effects.

The saturation profile is most likely varying along the core with a maximum at the production end, but since in-situ measurement of saturation was not possible, the average value was regarded as a reasonable estimate. At low IFT the capillary forces are very small and there will consequently be no capillary end-effect.

The Berea core used in all the depletion and displacement experiments, was relatively homogeneous and well consolidated, and with $50 \mathrm{~cm}$ length and $5.1 \mathrm{~cm}$ diameter. The porosity was measured to be as low as $15.3 \%$. Since this was considered too low for Berea, the measurements were repeated several times by injection of water and helium. The results were still almost the same.

The absolute permeability was determined to be 220 md both at reservoir and ambient conditions. With irreducible water present $\left(S_{w i}=25 \%\right)$, the permeability was reduced to $155 \mathrm{md}$. The irreducible water saturation was established by standard techniques, and reached approximately the same value each time.

Relative permeability measurements. Saturating the core by equilibrium liquid was a critical process. Though, by being extremely careful, it is anticipated that only small errors were made with respect to composition, saturation, etc. during the saturation process. The compositions of the first liquid production at each pressure step during the flow experiments were almost constant until breakthrough. This indicates that there is no discrepancy in the saturation prior to the start of the experiments. The compostions were almost identi$\mathrm{cal}$ to the corresponding compositions from the CVD-experiments.

Since injection of gas and liquid was performed with a positive displacement pump, giving a very accurate and constant flow, the flow conditions seemed to be very stable under most of the flow experiments. The measurements of $\Delta P$ showed that the differential pressure reached a stable value within 5 minutes. The back pressure system was operating smoothly, and no pressure fluctuation was observed.

A phase detector connected to the outlet of the core detected the breakthrough of the injection gas and gave also a fairly accurate reading of the flow rate of each phase in the two-phase region (proper calibration and known injection rate). The breakthrough was also detected by the pitoduction GOR, which rose sharply at the breakthrough. The two measurements gave approximately the same breakthrough when the time lag was taken into account.

Compositional analyses were performed on the produced liquid and gas. Based on these analyses and records of produced volumes, calculation of flow rates, interfacial tension etc., were performed as previously described'.

The calculation of relative permeability was based on the Welge method and on the steady state technique. In the first experiment $\left(P_{1}=19.20 \mathrm{MPa}\right.$ and the interfacial tension between gas 1 and liquid 1 (IFT) $=0.04 \mathrm{mN} / \mathrm{m}$ ) the relative permeabilities were almost straight lines. In the two other cases $\left(P_{2}=18.60 \mathrm{MPa}, \mathrm{FT}_{2}=0.14 \mathrm{mN} / \mathrm{m}\right.$ and $P_{3}=18.20 \mathrm{MPa}$, IFT $_{3}=0.26 \mathrm{mN} / \mathrm{m}$ ), the relative permeabilities curved more and were significantly reduced with increasing interfacial tension. For the repetition of this series, the results were almost the same with the following results: for the lowest IFT $\left(\right.$ IFT $_{4}=0.03 \mathrm{mN} / \mathrm{m}, P_{4}=19.26 \mathrm{MPa}$ ) the relative permeabilities were straight lines. In the two other cases $\left(P_{5}=18.65 \mathrm{MPa}\right.$, IFT $_{5}=0.12 \mathrm{mN} / \mathrm{m}$ and $P_{6}=18.15 \mathrm{MPa}, \quad \mathrm{IFT}_{6}=0.29 \mathrm{mN} / \mathrm{m}$ ) the rel.perm. curves were reduced with increasing IFT and curved more. These observations have been detected by other researchers (referred earlier in this paper). This states that effects of interfacial tension on relative permeability must be taken into consideration in evaluation of gas condensate reservoirs.

The error made by changing the flow ratios of liquid and gas before a true steady state condition was reached might be a little bit difficult to estimate accurately. Since the mobility of the two phases is a function of their own saturation (straight lines) at low IFTs, the error is anticipated to be the same as for the determination of flow rates for each phase. Since also no specific problem with accumulation of liquid or gas was observed, we feel certain that this simplification is justified.

As can be seen for the more simple reference systems, similar results can be obtained, and the observations consequently apply not only to or gas condensate systems very close to the critical point, but also to non-critical systems with somewhat higher IFT. 
Another important observation to make is the very low residual saturations obtained for all experiments. Even for the highest values of IFT (0.29 $\mathrm{mN} / \mathrm{m}$ ) with relatively curved rel. perms, the residual saturation is less than 10 percent. If the two displacements had been carried out for more than 4 pore volumes, there might have been some more recovery of liquid with correspondingly lower residual saturations.

The steady state technique was only used for the lowest IFT experiments. If a displacement had been performed at this pressure, little two-phase flow would have taken place with similarly few experimental points. This was the major reason for why we did not use displacement in all cases.

We tried to make spreading and non-spreading systems, but this was not easy. We never obtained non-spreading systems robust enough to account for a non-spreading property during the flow experiments.

\section{CONCLUSIONS}

The flow experiments showed a strong relationship between relative permeability and interfacial tension of the gas and condensed liquid system investigated. At the first experimental pressure $(P=19.2 \mathrm{MPa}$ and $\mathrm{IFT}=0.03 \mathrm{mN} / \mathrm{m}$ ) the relative permeabilities were almost straight lines (linear diagram). For the two other pressures ( $\mathrm{P}=18.6$ $\mathrm{MPa}$, IFT $=0.13 \mathrm{mN} / \mathrm{m} ; \mathrm{P}=18.2 \mathrm{MPa}, \quad$ FTT $=0.27$ $\mathrm{mN} / \mathrm{m}$ ), the relative permeabilities curved more and were significantly reduced with increasing interfacial tension. This states that effects of interfacial tension on relative permeability, must be taken into consideration in evaluation of gas condensate reservoirs.

Residual saturations of condensate (and thus also critical saturation for flow of condensate) are very low for the whole range investigated. This implies that the residual saturations may be low even at IFTs as high as $1 \mathrm{mN} / \mathrm{m}$. Though, this need more work before more solid conclusions can be drawn.

\section{NOMENCLATURE}

$\mathrm{k}_{\mathrm{g}}=$ effective permeability to gas, ind

$\mathrm{k}_{\mathrm{o}}=$ effective permeability to oil, $\mathrm{md}$

$\mathrm{k}_{\mathrm{rg}}=$ relative permeability to gas

$\mathrm{k}_{\mathrm{r}}=$ relative permeability to oil $\mathrm{p}=$ total pressure, $\mathrm{MPa}$

$\mathrm{Pc}=$ capillary pressure, $\mathrm{kPa}$

$\Delta \mathrm{p}=$ pressure drop, $\mathrm{Pa}$

$\mathrm{S}_{\mathrm{g}}=$ gas saturation

$S_{m}=$ residual or irreducible water saturation

$\mathrm{S}_{\mathrm{ax}}=$ residual oil saturation

$\mu=$ viscosity, $\mathrm{cP}$

$\mathbf{R}=$ capillary radius, $\mathbf{m}$

$\sigma=$ interfacial tension, $\mathrm{mN} / \mathrm{m}$

$\rho=$ density, $\mathrm{kg} / \mathrm{m}^{3}$

$\mathrm{u}=$ velocity, $\mathrm{m} / \mathrm{s}$

$\mathrm{N}_{\mathrm{B}}=$ Bond number

$\mathrm{N}_{\mathrm{c}}=$ Capillary number

$\mathrm{Re}=$ Reynolds number

\section{ACKNOWLEDGMENTS}

The work presented in this paper was performed on funding from SPOR, the Norwegian State R\&D Program for Improved Oil Recovery and Reservoir Technology, and the equipment was sponsored by Saga Petroleum and Statoil. Some of the work was also performed as a part of a Scholarship given by the Research Council of Norway. Finally, we will thank our colleagues for invaluable help during the performance of the work on which this publication is based.

\section{REFERENCES}

1. MUNKERUD, P.K: "Measurements of Relative Permeability and Flow Properties of a Gas Condensate System During Pressure Depletion and Maintenance", Paper SPE 19071, presented at the SPE Gas Technology Symposium, June 7-9, 1989, Dallas, Texas, USA.

2. BJØRKVIK, B.J.A, STRAND, K.A, STRØMME, G., SIKKELAND, T., WAALER, D., MUNKERUD, P.K, AKERVOLL, I. AND SOLHEIM, A., 1994: "Description of an interface light scattering spectrometer with a cylindrical high pressure scattering cell including performance test on a model gas condensate system", Journal of Colloid and Interface Science, 164 (1994), 151-62.

3. HOUGH, E.W. and STEGEMEIER, G.L.: "Correlation of Surface and Interfacial Tension of Light Hydrocarbons In the Critical Region", Soc.Petr.Eng.J., Dec. 1961, 259-263. 
4. WEINAUG, C.F and KATZ, D.L.: "Surface Tensions of Methane-Propane Mixtures", Ind. Eng. Chem. (1943) 35, 239.

5. BARDON, C. and LONGERON, D.G.: "Influence of very low Interfacial Tension on Relative Permeability", Soc.Petr.Eng.J., October 1980, 391-401.

6. BARBIAUX, B.J., and LIMBORG, S.G.: "An Intergrated Experimental Methodology for a Better Prediction of Gas Condensate Flow Behaviour". Paper SPE 28931, presented at the SPE 1994 Annual Conference and Exhibition, New Orleans, LA, September 28-30, 1994.

7. ASAR, H. and HANDY, L.L.: "Influence of Interfacial Tension on Gas-Oil Relative Permeability in a Gas Condensate System": Paper SPE 11740, presented at the SPE 61st Annual Technical Conference and Exhibition, 11p, 1983.

8. SAEIDI, A. and HANDY, L.L.: "Flow and Phase Behaviour of Gas Condensate and Volatile Oils in Porous Media". Paper SPE 4891, presented at the fifth SPE Annual California Regional Meeting, San Francisco, Calif., April 4-5, 1974, 12 p.

9. DUMORE, J.M. and SCHOLS, R.S:: "Drainage Capillary Pressure Functions and the Influence of Connate Water". Soc.Petr.Eng.J., October 1974, pp. 437-444
10. KING, R.L. and STILES, J,H!" "A Reservoir study of Hawkins Woodbine Field". Paper SPE 2972 presented at the SPE/AIME Fall Meeting, Houston, Oct. 4-7, 1970.

11. HAGOORT, J.: "Oil Recovery by Gravity Drainage". Soc.Petr.Eng.J., June 1980, pp. 139150.

12. DELCLAUD, J., ROCHOU, J. and NECTOUX, A.: "Investigation of Gas/Oil Relative Permeabilities: High Permeability Oil Reservoir Application", Paper SPE 16966 presented at the SPE 62nd Annual Technical Conference and Exhibition, Dallas, Texas, September $27-30,1987,339-352$.

15. HUSTAD, O.S.: CERES - An EOS Compositional Reservoir Simulator. IKU Petroleum Research in-house simulator, Version 10. (July, 1994).

16. HOLT, T., HUSTAD, O.S. and SKURDAL, H.: Film Flow Mechanisms During Gas Flooding. Paper presented at the Fourteenth IEA Collaborative Project on Enhanced Oil Recovery Workshop and Symposium, Salzburg (Oct. 17-21, 1993). 